\title{
The Characters of a Mid-aged Patient COVID-19 Infected After Nasopharyngeal Swab PCR Assay Negative for 14 Times A Case Report
}

\author{
Juan Yang ${ }^{1}$, Xiaohui Zhao ${ }^{1}$, Wanju Sun ${ }^{3}$, Yongbing Wang ${ }^{3}$ and Haijing Sui ${ }^{2 *}$ \\ ${ }^{1}$ Department of Neurology, Shanghai Pudong New Area People's Hospital Affiliated to Shanghai University of Medicine \& \\ Health Sciences, Shanghai, People's Republic of China
}

${ }^{2}$ Department of Image, Shanghai Pudong New Area People's Hospital Affiliated to Shanghai University of Medicine \& Health Sciences, Shanghai, People's Republic of China

${ }^{3}$ Shanghai Pudong New Area People's Hospital, China

*Corresponding author: Haijing Sui, Department of Image, Shanghai Pudong New Area People's Hospital Affiliated to Shanghai University of Medicine \& Health Sciences, Shanghai, People's Republic of China.

\section{ARTICLE INFO}

Received: 彗 June 27, 2020

Published: 㗀 July 06, 2020
ABSTRACT

Citation: Juan Yang, Xiaohui Zhao, Wanju Sun, Yongbing Wang, Haijing Sui. The Characters of a Mid-aged Patient COVID-19 Infected After Nasopharyngeal Swab PCR Assay Negative for 14 Times A Case Report. Biomed J Sci \& Tech Res 28(4)-2020. BJSTR. MS.ID.004689.

\section{Case Report}

A 40-year-old man without any medical history and any symptom presented to the COVID-19-screening-room of emergency with complaint contacting with a patient COVID-19 infected for 3 hours, who came from Wuhan by airplane for his vacation and happen to seated beside a person with cough who was confirmed COVID-19 infected at later. He was tracked by CDC staff after 4 hours later departed from airport and was informed to screen the virus in our hospital. On presentation, the patient was afebrile with normal vital signs. A nasopharyngeal swab was sent to test COVID-19 by Polymerase Chain Reaction (PCR) assay, which was negative. Blood routine analysis showed a high red cell distribution width (RDW) (reference range 11.6-14), low Platelet Distribution Width(PDW) (reference range 15.1-18.1), low Mean Platelet Volume (MPV) (reference range 9.4-12.5), high monocyte (\%)(reference range 3-10) and the other indexes were normal. Chest computed Tomography (CT) demonstrated a lesion in right superior lobe of lung described as latticed ground glass sign (Figure 1).

The man was isolated in observation room in hospital for observation of symptoms and vital signs every day. The chest CT was scanned at 48-hour interval and nasopharyngeal swab for COVID-19 was tested at 24-hour interval. At the sixth day, he had (temperature $37.8^{\circ} \mathrm{C}$ ) and diarrhea. The symptoms were worse persistently. The results of chest CT revealed that the number of the lung lesion increased continuously since the first test, but the results of COVID-19 test were negative still in each testing. After two days of having fever, the temperature was $39.8^{\circ} \mathrm{C}$ the lung lesion progressed to large lesion in two lobes without clearly numbers (Figure 1). Given the patient's fever symptom in combination with lung CT deteriorative, he was started on Arbidol hydrochloride( $0.2 \mathrm{~g}$ tid, po), Immunoglobulin(5g qd, intravenous infusion) and Thymosin (1.6mg biw, intravenous infusion), although COVID-19 test was negative. At the fourth day after taking medicine, the symptom of fever and diarrhea were vanished. But the large lesion of the lung was persisted. Blood routine analysis showed that except the abnormal of RDW, PDW, MPV and monocyte (\%) persisting, eosinophils\% was low (reference range 0.4-8).

The results of COVID-19 test were negative still. At the tenth day after no fever, the large lesion of the lung took a turn to better (Figure 1). Blood routine analysis showed that the abnormal of RDW, PDW, MPV and monocyte (\%) persisting, but eosinophils\% was normal. The COVID-19 test was negative still. He was advised to persist taking Arbidol hydrochloride only and discharge from hospital with advices of isolating at home for 14 days and come back for retested lung CT and COVID-19 then. The fourteenth day after discharging, 
the patient come back to retested chest CT and COVID-19. The chest CT was normal. The COVID-19 was positive eventually thorough serum antibody by enzyme-linked immunosorbent assay (ELISA) after nasopharyngeal swab PCR assay negative for 14 times.

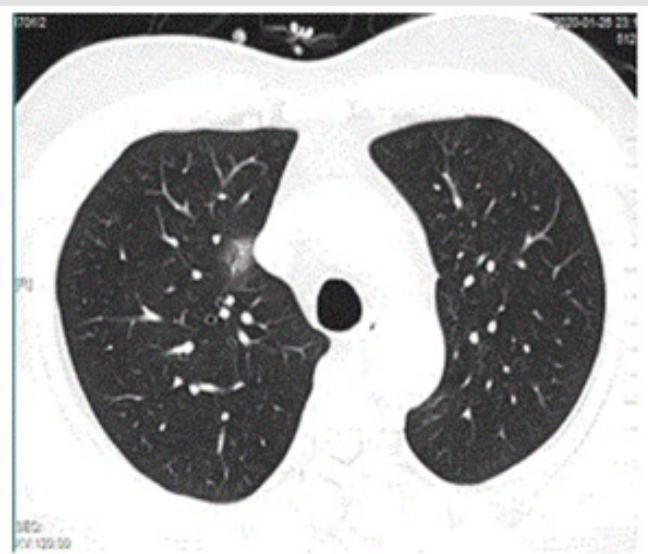

A

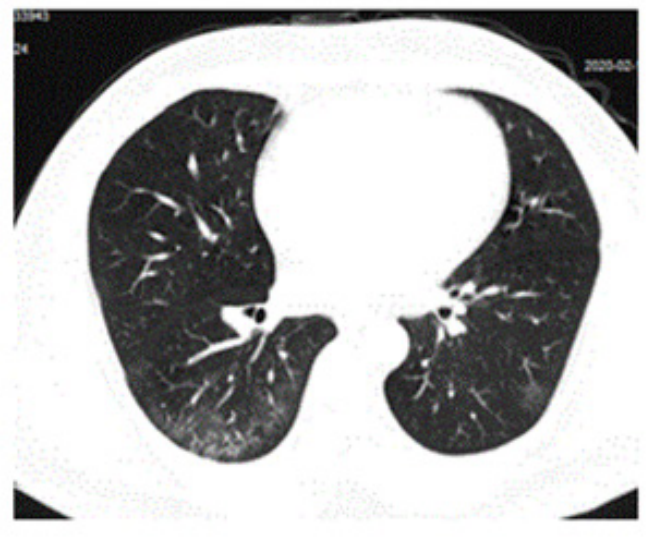

C

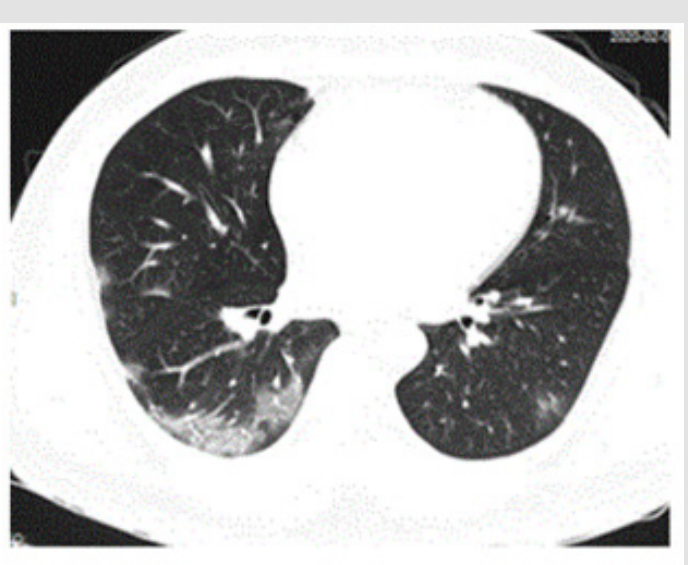

B

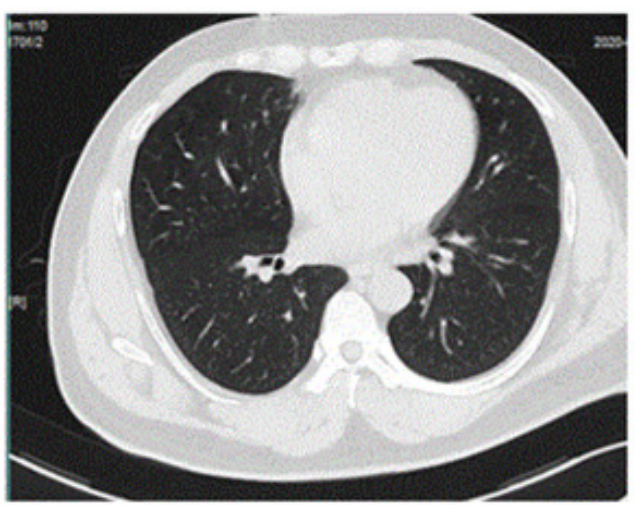

D

Figure 1: The different CT imaging of the patient COVID-19 infected in different time. A was CT imaging of the patient when presented; B was CT imagingof the patient when the temperature was highest; C was CT imaging of the patient the tenth day after fever vanish; D was CT imaging of the patient the fourteenth day after discharging.

\section{Discussion}

This patient was diagnosed as COVID-19 infected by serum antibody ELISA assay eventually after being negative 14 times by nasopharyngeal swab PCR assay, which indicated the low positive rate of nasopharyngeal swab PCR assay, that were coincided with another Chinese study [1]. This was a patient with no symptom and COVID-19 testing negative at presentation, but he had the history of contacting with the patient COVID-19 infected and the lung CT was with one latticed ground glass sign in right superior lobe. It indicated that lung impairment may started before clinical symptoms. Retrospective analysis, it was suggested strongly that the history of contacting COVID-19 patient and the characters lesion of lung CT were very important for diagnosis of COVID-19 infected, that were coincided with the other study [2,3]. The blood routine analysis demonstrated low eosinophils\% was presented when lung impaired most seriously and returned to normal at the time lung impairment got better obviously, which suggest that eosinophils\% may be the marker of the severity of the disease. At the fourth day after taking medicine, the symptom of fever and diarrhoea were vanished but the large lesion of the lung persisted. It indicated that the earlier drug intervention may induce faster recovery of midaged COVID-19 infected without any disease history. We recognize the limitations of this single case report and more evidence needed to be find in more cases.

\section{References}

1. Ma SY, Luo YM, Hu TY, You ZC, Sun JG, et al. (2020) Clinical application effect of modified nasopharyngeal swab sampling for 2019 novel coronavirus nucleic acid detection. Zhonghua Shao Shang Za Zhi 36(0).

2. Li Y, Xia L (2020) Coronavirus Disease 2019 (COVID-19): Role of Chest CT in Diagnosis and Management. AJR Am J Roentgenol 214(6): 12801286.

3. Rubin GD, Ryerson CJ, Haramati LB, Sverzellati N, Kanne JP, et al. (2020) The Role of Chest Imaging in Patient Management during the COVID-19 Pandemic: A Multinational Consensus Statement from the Fleischner Society. Chest 3692(20): 30673-30675. 
ISSN: 2574-1241

DOI: $10.26717 / B J S T R .2020 .28 .004689$

Haijing Sui. Biomed J Sci \& Tech Res

(C) (9) This work is licensed under Creative

Submission Link: https://biomedres.us/submit-manuscript.php

$\begin{array}{ll}\text { BIOMEDICAL } & \text { Assets of Publishing with us } \\ \text { RESEARCHES } & \text { - Global archiving of articles } \\ \text { - Immediate, unrestricted online access } \\ \text { - Rigorous Peer Review Process } \\ \text { https://biomedres.us/ }\end{array}$

